ESJ Social Sciences

\title{
Droits des Enfants dans le Programme Scolaire au Primaire en Cote d'Ivoire et Leurs Perceptions par les Enseignants
}

\author{
Ouattara Kanndanan Insiata
}

Ecole Normale Supérieure d'Abidjan, Cote d'Ivoire

Doi:10.19044/esj.2021.v17n35p263

Submitted: 12 August 2021

Accepted: 11 October 2021

Published: 31 October 2021
Copyright 2021 Author(s)

Under Creative Commons BY-NC-ND

4.0 OPEN ACCESS

Cite As:

Insiata O.K. (2021). Droits des Enfants dans le Programme Scolaire au Primaire en Cote d'Ivoire et Leurs Perceptions par les Enseignants. European Scientific Journal, ESJ, 17 (35), 263. https://doi.org/10.19044/esj.2021.v17n35p263

\section{Résumé}

Le 20 novembre 1989, l'Assemblée Générale de l'Organisation des Nations unies a adopté, à l'unanimité, la Convention relative aux droits de l'enfant . Une éducation aux droits de l'enfant semble être alors essentielle, en ce sens qu'elle permet à l'enfant en tant qu'être vulnérable et dépendant, d'acquérir des connaissances sur ces droits. Ainsi, cette étude se propose d'une part, de mettre en exergue les contenus d'enseignements relatifs au thème des droits de l'enfant dans le programme scolaire de l'école primaire en Côte d'Ivoire. D'autre part, elle met en relation le contenu de cet enseignement et les représentations des instituteurs interrogés à ce sujet. Une analyse de contenu des guides maître et des entretiens semi directifs réalisés avec 12 instituteurs montrent que, si les droits des enfants sont pris en compte dans le curriculum formel, nous notons une divergence de point de vue dans la perception chez ceux-là même qui sont chargés de les dispenser.

Mots clés: Droit de l'enfant, Guide-maître, Perception, Enseignant, Ecole primaire 


\title{
Children's Rights in the Primary School Program in Cote d'Ivoire and Their Perceptions by Teachers
}

\author{
Ouattara Kanndanan Insiata
}

Ecole Normale Supérieure d'Abidjan, Cote d'Ivoire

\begin{abstract}
On November 20, 1989, the United Nations General Assembly unanimously adopted the Convention on the Rights of the Child. Education of the child's rights seems, therefore, to be very essential because it helps the child, as a vulnerable and dependent being, to acquire knowledge about these rights. This paper focuses, on the one hand, on highlighting the teaching content relating to the theme of the rights of the child in the school curriculum of primary school in Côte d'Ivoire. On the other hand, it focuses on the content of this teaching and the representations of the teachers questioned in this subject. A content analysis of the master guides and of the semi-structured interviews carried out with 12 teachers show that if the rights of children are taken into account based on the formal curriculum, a divergence of viewpoint would be mentioned through the perceptions of those who are even in charge of teaching them.
\end{abstract}

Keywords: Children's rights, Master guide, Perception, Teacher, Primary school

\section{Introduction}

En accédant à la souveraineté nationale en 1960, la Côte d'Ivoire a eu comme priorité, la valorisation des ressources humaines sans laquelle aucun développement endogène n'est possible. Cette valorisation passe nécessairement par l'éducation et la formation de ses enfants. Or, l'idée que l'enfant est un être vulnérable qu'il faut protéger et lui garantir des droits spécifiques et les faire respecter, a conduit à l'adoption de la Convention Internationale des Droits de l'Enfant (CIDE). ${ }^{1} 191$ pays y compris la Côte d'Ivoire $^{2}$, ont signé et ratifié cette convention dont les quatre principes fondamentaux sont: la non-discrimination, la priorité donnée à l'intérêt supérieur de l'enfant, le droit de vivre, de survivre et de se développer, le respect des opinions de l'enfant.

${ }^{1}$ La Convention internationale des droits de l'Enfant (CIDE) a été adopté le 20 novembre 1989 par l'Assemblée Générale de l'Organisation des Nations Unies.

${ }^{2}$ La Côte d'Ivoire a ratifié le 04 février 1991 la Convention Internationale des Droits de l'Enfant. 
Le droit à l'éducation ${ }^{3}$ en tant que droit fondamental stipule que tout enfant a le droit d'aller à l'école et d'avoir accès à des connaissances qui l'aideront à préparer sa vie d'adulte. Dès lors, le droit à l'éducation semble être une question importante pour toutes les sociétés. L'UNESCO ${ }^{4}$ considère, que l'éducation est l'un des outils les plus puissants pour sortir de la pauvreté les enfants et les adultes socialement exclus, et faciliter leur insertion dans la société. Cette institution affirme que si tous les adultes achevaient leur cycle d'études secondaires, le nombre de personnes touchées par la pauvreté dans le monde pourrait être divisé par deux, sinon plus 5 . Or, En 2018, Selon ISU ${ }^{6}$, $8,2 \%$ des enfants en âge d'aller à l'école primaire ne sont pas scolarisés. Seuls six jeunes sur dix termineront leurs études secondaires en 2030. Le taux d'alphabétisation des jeunes (15-24 ans) est de 91,73\%, ce qui signifie que 102 millions de jeunes n'ont pas les compétences de base en lecture et en écriture. Si on s'intéresse au système éducatif ivoirien, le rapport 2019-2020 du PASEC ${ }^{7}$, indique que $66,9 \%$ des apprenants ne disposent pas de compétences leur permettant de poursuivre sans difficultés leurs apprentissages.

Conscient que l'éducation est l'un des investissements les plus importants qu'un pays puisse faire pour son avenir, la loi $\mathrm{n}^{\circ} 2016-886 \mathrm{du} 8$ novembre 2016 portant Constitution de la République de Côte d'Ivoire a, en son article 10 et les différents textes législatifs et règlementaires, qui en définissent les modalités d'application, affirmé que «l'école est obligatoire pour tous les enfants des 2 sexes dans les conditions déterminées par la loi ». D'ailleurs, les autorités ivoiriennes ont institué la gratuite de l'éducation et fait adopter la politique de l'école obligatoire ${ }^{8}$ qui confère le droit à tous les enfants de six (6) et seize (16) ans d'accéder et de poursuivre une scolarité. La mise en œuvre des dispositions ${ }^{9}$ de cette politique devrait à terme faciliter le

${ }^{3}$ Le droit à l'éducation est l'article 18 de la convention des droits de l'enfant.

${ }^{4}$ Organisation des Nations Unies pour l'Education, la Science et la Culture.

${ }^{5}$ Extrait du document d'orientation, intitulé «Réduire la pauvreté mondiale par l'enseignement primaire et secondaire universel » de juin 2017

${ }^{6}$ Consulté sur le https://fr.unesco.org/news/ce-que-vous-devez-savoir-droit-leducation

${ }^{7}$ Programme d'Analyse de Systèmes Éducatifs (PASEC), produit régulièrement des rapports d'évaluation sur les compétences de bases des élèves du primaire dans différents systèmes éducatifs en Afrique.

${ }^{8} \mathrm{~L}$ 'école obligatoire intervient suite au projet de loi portant modification de la loi $\mathrm{N}^{\circ} 95-696$ du 07 septembre 2015 relative à l'enseignement.

${ }^{9}$ Les grandes articulations de cette nouvelle loi concernent la réforme du système éducatif ivoirien, les pesanteurs sociologiques et religieuses, la proximité des écoles des lieux de résidences, le rôle des collectivités territoriales, la prise en compte des langues nationales dans le programme scolaire, les dispositions prévues à l'encontre des enseignants fondateurs d'établissements scolaires privés, le manque d'infrastructures, de matériels didactiques et de personnel enseignant, la distribution tardive des kits scolaires gratuits, les conditions de vie des enseignants et le déclin du système éducatif 
déroulement du cursus scolaire c'est-à-dire l'accès, le maintien et la réussite à l'école, en tant qu'espace de promotion des droits de l'enfant. Etudier les droits de l'enfant dans le contexte scolaire, peut être jugé comme un sujet d'actualité dont certains aspects qui le justifie ont été relevé par Zermatten $(2004)^{10}$. Si, l'une des missions de l'enseignement est de former les élèves à la citoyenneté, le traitement de la thématique des droits de l'enfant dans les classes pourrait contribuer à développer l'esprit critique de ceux-ci en tant que citoyens de demain. En travaillant sur cette problématique, la préoccupation centrale est de mettre en évidence les notions des droits de l'enfant intégrées dans les contenus l'éducation de base notamment au primaire. Cette étude se propose également d'analyser les perceptions des instituteurs chargés de les enseigner et de les promouvoir dans le contexte de la classe.

\section{Methodes}

Le terrain de l'étude dans la circonscription de l'Enseignement Préscolaire et Primaire de Yopougon Maroc qui couvre au total 51 écoles dont deux (2) ont été choisies de façon aléatoire. Il n'est nullement question de généraliser les résultats de ce travail au reste du pays mais plutôt d'apporter une contribution à la compréhension de l'enseignement des droits de l'enfant en Côte d'Ivoire. Pour la collecte des données, la méthode qualitative a été adoptée. En effet, il a été procédé, à une analyse de document, précisément des guides maîtres dans la discipline Education aux Droits de l'Homme et à la citoyenneté (EDHC) qui prennent en compte les leçons relatives aux droits de l'enfant. En outre, dans le but de recueillir les perceptions, douze (12) enseignants de tous les niveaux de l'ordre de l'enseignement du primaire ont été soumis à des entretiens individuels semi directifs enregistrés sur des supports magnétiques. Les questions du guide d'entretien auxquelles ils ont répondu sont entre autres : Quelles sont vos perceptions vis à vis des droits des enfants en général? Que pensez-vous de leur prise en compte dans le curriculum du primaire ? Quels aspects sont-ils pris en compte ? quels sont les aspects à approfondir ? Ces entretiens ont été choisis car comme le souligne Patton (2002), ils aident à accéder aux pensées de quelqu'un et à l'ampleur de ces dernières, ce qui cadre bien avec le deuxième objectif de cette étude relatif à la perception des enseignants. L'analyse de contenu des données obtenues grâce aux retranscriptions des entretiens semi-directifs, a permis de relever les points de convergence et divergence.

${ }^{10}$ Zermatten (2004, pp. 195-196). 


\section{Resultats et Discussion}

\section{La Prise en compte des thèmes relatifs aux droits de l'enfant dans le curriculum du primaire}

L'enjeu majeur pour toute école respectueuse des droits de l'enfant est de s'assurer que tous les apprenants disposent de droits, qu'ils soient codifiés dans la Déclaration universelle des droits de l'homme, ou dans la Convention Internationale des Droits de l'Enfant (CIDE), ou toute autre convention applicable. La question du curriculum au sein de l'institution scolaire qui porte le plus souvent sur les savoirs transmis est important, puisque l'école est le lieu où se forgent les valeurs humaines indispensables pour le développement harmonieux d'une nation. Il faut noter que lorsqu'on parle de curriculum, l'aspect le plus évident est le débat récurrent sur les « programmes scolaires » à savoir ce qui doit être enseigné, comment l'enseigner et à quel niveau. Le curriculum étant perçu comme un construit social, qui traduit des postures idéologiques et des rapports de pouvoir, certains auteurs Nozaki et Apple (2002), puis Apple (2006) considèrent que sa mise en place aboutit systématiquement à la construction d'un curriculum caché, dans la mesure où le curriculum est toujours une sélection de savoirs sélectionnés par des groupes d'intérêt en situation de pouvoir dans la société. Le recours aux guides maitres de l'école primaire, montre bien la volonté des autorités éducatives ivoiriennes, de prendre en compte les droits de l'enfant dans le choix du curriculum à enseigner. En effet, pour une offre éducative inclusive, équitable et de qualité, l'Unesco a élaboré la stratégie pour la santé et le bienêtre dans le cadre de son mandat international de coordination et de suivi de l'ODD4 $^{11}$ à travers l'Agenda Education 2030 ${ }^{12}$. C'est une exigence qui repose sur le droit à une éducation complète à la sexualité. Selon une enquête ${ }^{13}$, ce sont 5076 cas d'élèves en grossesse dans le primaire avec un âge minimum de 9 ans et au niveau scolaire de CE2 qui ont été enregistrés au cours de l'année scolaire 2012-2013 contre 1292 en 2007-2008, soit une augmentation annuelle de $41 \%$ en moyenne. En prenant donc en compte un module sur l'éducation complète à la sexualité, cela peut être considéré comme une réponse à plusieurs facteurs d'abandon scolaire et de disparité de genre dans l'éducation du fait des grossesses en cours de scolarité. Toutefois, ce module qui est en

\footnotetext{
${ }^{11}$ ODD4 : «Assurer l'accès de tous à une éducation de qualité, sur un pied d'égalité, et promouvoir les possibilités d'apprentissage tout au long de la vie ». Cet objectif vise donc à garantir à tous et à toutes l'accès à une éducation équitable, gratuite et de qualité à travers toutes les étapes de la vie en éliminant notamment les disparités entre les sexes et revenus. Il met également l'accent sur l'acquisition de compétences fondamentales et de niveau superieur pour vivre dans une société durable.

${ }^{12}$ En septembre 2015, les 193 États membres de l'ONU ont adopté le programme de développement durable à l'horizon 2030, intitulé Agenda 2030. C'est un agenda pour les populations, pour la planète, pour la prospérité, pour la paix et par les partenariats.

${ }^{13}$ Enquête du ministère en charge de l'éducation nationale sur les grossesses en milieu scolaire
} 
phase d'expérimentale n'est pas encore généralisé bien qu'il vise à apporter aux apprenants les connaissances, les compétences, les attitudes et les valeurs qui leur permettront d'avoir une vision positive de leur sexualité dans le cadre de leur développement émotionnel et social. Par ailleurs, lorsqu'on parcourt le guide du maître dans la discipline EDHC, nous y notons l'inscription de plusieurs leçons sur les droits de l'enfant dans les différents niveaux d'enseignement comme illustrées dans le tableau ci-dessous.

Tableau 1. Les leçons sur les droits de l'enfant et les objectifs attendus en fonction du niveau d'étude dans le primaire en Côte d'Ivoire

\begin{tabular}{|c|c|c|}
\hline $\begin{array}{l}\text { Niveau } \\
\text { d'étude }\end{array}$ & Leçons & Consignes pédagogiques \\
\hline $\mathrm{CP} 1$ & $\begin{array}{l}\text { Les Droits de } \\
\text { l'enfant }\end{array}$ & $\begin{array}{l}\text {-Amener les enfants à comprendre que chaque enfant a } \\
\text { droit à un nom et un prénom; } \\
\text {-Amener les enfants à comprendre que nous sommes tous } \\
\text { égaux malgré la différence et la diversité de nos noms. }\end{array}$ \\
\hline $\mathrm{CP} 2$ & $\begin{array}{l}\text { Le droit à la } \\
\text { différence. }\end{array}$ & $\begin{array}{l}\text { Amener les apprenants à comprendre que le respect de } \\
\text { l'autre et de ses différences permet de prévenir l'extrême } \\
\text { violence et d'être un bon citoyen : } \\
\text {-éviter les injures, les humiliations, les offenses ; } \\
\text { - respecter la dignité des autres ; } \\
\text { - respecter la liberté, l'égalité, le genre, } \\
\text { - amener les apprenants à donner leur avis sur des } \\
\text { décisions qui les concernent. }\end{array}$ \\
\hline \multirow[t]{2}{*}{ CE1 } & $\begin{array}{l}\text { L1 : Le droit à la } \\
\text { nationalité. }\end{array}$ & $\begin{array}{l}\text { Amener les apprenants à faire ressortir l'importance du } \\
\text { droit à la nationalité, en être fier et respecter celle des } \\
\text { autres pour éviter l'extrême violence. }\end{array}$ \\
\hline & $\begin{array}{l}\text { L2: Les droits à } \\
\text { l'éducation et à la } \\
\text { santé et le bien-être } \\
\text { de l'enfant. }\end{array}$ & $\begin{array}{l}\text { Amener les apprenants à comprendre que la violence } \\
\text { trouve souvent ses sources dans le manque d'éducation et } \\
\text { le citoyen éduqué est mieux outillé pour éviter l'extrême } \\
\text { violence. } \\
\text { - Amener les apprenants à comprendre }\end{array}$ \\
\hline \multirow[b]{2}{*}{ CE2 } & $\begin{array}{l}\text { Le droit à la } \\
\text { différence et le bien- } \\
\text { être de l'enfant }\end{array}$ & $\begin{array}{l}\text {-Amener les apprenants à accepter l'autre avec ces } \\
\text { différences religieuses, ethniques, physiques sociales. } \\
\text { Amener les apprenants à comprendre l'importance } \\
\text { d'accepter l'autre avec ses différences évite l'extrême } \\
\text { violence. }\end{array}$ \\
\hline & $\begin{array}{l}\text { Notion de la liberté } \\
\text { d'expression }\end{array}$ & $\begin{array}{l}\text { Le droit pour toute personne de penser comme elle le } \\
\text { souhaite et de pouvoir exprimer ses opinions. } \\
\text { - La liberté de donner son avis sur tous les sujets } \\
\text { - La liberté d'association, de réunion... }\end{array}$ \\
\hline CM1 & $\begin{array}{l}\text { L } 1: \text { Les droits et } \\
\text { les devoirs. }\end{array}$ & $\begin{array}{l}\text {-Amener les apprenants à comprendre que tous les } \\
\text { Hommes ont les mêmes Droits. } \\
\text { - Avoir les mêmes droits signifie que l'Etat doit pourvoir } \\
\text { à nos besoins de santé, d'éducation, de participation et de } \\
\text { protection dans l'équité ; } \\
\text { - respecter les Droits de l'autre est de comprendre que nos } \\
\text { droits ont des limites; }\end{array}$ \\
\hline
\end{tabular}




\begin{tabular}{|c|c|l|}
\hline \multirow{2}{*}{} & $\begin{array}{c}\text { L 2 : Le droit à la } \\
\text { protection de } \\
\text { l'enfant }\end{array}$ & $\begin{array}{l}\text { - assumer ses obligations, c'est de contribuer au } \\
\text { développement de son pays. }\end{array}$ \\
\cline { 2 - 3 } & $\begin{array}{l}\text { Amener les apprenants à initier des actions de promotions } \\
\text { de leur droit à la protection à travers leur participation } \\
\text { active aux activités de vie scolaire. }\end{array}$ \\
\hline CM2 & $\begin{array}{c}\text { Les Droits à la } \\
\text { participation de } \\
\text { l'enfant. }\end{array}$ & $\begin{array}{l}\text {. Amener les apprenants à comprendre qu'ils ont } \\
\text { l'obligation de participer aux prises de décisions pour } \\
\text { éviter l'extrême violence }\end{array}$ \\
\hline
\end{tabular}

Source : Auteur elle-même (2021), données tirées des guides du maitre

Nous constatons que les contenus en Education aux Droits de l'Homme et à la Citoyenneté portent sur le droit à la vie, le droit à une nationalité, le droit à la protection, les droits et les devoirs et aussi le célèbre article 12 de la convention des droits de l'enfant qui stipule le droit à exprimer librement son opinion. Cette dernière étant dûment prise en considération pour toute question concernant l'apprenant. Nous nous sommes cependant posé la question de savoir ce qu'en pensent les acteurs chargés de dispenser ces leçons ?

\section{Perceptions des droits des enfants dans le curriculum par les enseignants}

Il a été question dans un premier temps de savoir ce que les enseignants du primaire pensent de la prise en compte des droits des enfants dans les contenus enseignés. Cette question semble importante, car la théorie sociale cognitive de Bandura (1997) souligne l'importance que jouent les systèmes d'attentes dans les décisions de s'engager ou non dans une action déterminée. Un enseignant dans sa pratique, doit amener les élèves vers la connaissance. Or, la pratique enseignante peut être influencée par la manière d'interpréter et de penser la "réalité », le contexte. Lorsque nous avons procédé à l'analyse de contenu des entretiens, il ressort que les enseignants interrogés évoquent plusieurs éléments dans leurs perceptions concernant la prise en compte des droits des enfants en tant que leçons enseignées à travers EDHC.

D'un côté, il y a ceux qui estiment que c'est un avantage que les élèves possèdent des connaissances sur leurs droits dès leur première année de scolarité. «Inculquer à l'enfant le respect des droits de l'homme et des libertés fondamentales $\gg$ me semble très important selon cette institutrice. D'ailleurs, certains considèrent que la « journée internationale des droits de l'enfant» qui a lieu chaque année le 20 novembre, est une preuve manifeste de cette importance. Si cette autre enseignante relève que cela répond à « une nécessité d'ouvrir les élèves au monde », cet autre interviewé quant à lui, affirme que non seulement le droit pour toute personne de penser comme elle le souhaite et de pouvoir exprimer ses opinions est enseigné, mais que son application 
dans le contexte classe est une réalité. En effet, La stratégie pédagogique utilisée au cours des leçons à travers le jeu de rôle, qui dramatisent la leçon étudiée permet de mieux assoir la connaissance. "A partir de la Classe de cours Elémentaire (CE), les élèves sont libres de former des groupes. Ils choisissent eux même un chef qui est leur porte-parole, un rapporteur et chacun s'exprime au sein du groupe, lors des travaux » atteste cet enseignant de la classe de CE2. L'importance de consacrer du temps à la participation des enfants a été souligné par plusieurs chercheurs (Tisdall, 2015b ; Spyrou, 2011 ; Lansdown, 2001). Les enfants doivent être encouragés à donner leur opinion même si celle-ci n'est pas identique à l'avis de l'adulte (Lansdown, 2010). En outre, dans ce groupe d'enquêtés, certains ont une perception négative de l'enseignement de l'éducation complète à la sexualité, qui ne cadre pas avec le contexte africain car la pudeur est au cœur des valeurs selon eux. On pourrait alors se poser la question de savoir comment un enseignant dispensera un cours sur la sexualité avec une telle perception.

D'autres enquêtés par contre, estiment qu'un accent particulier devrait être mis sur les devoirs au dépend des droits des enfants. Selon eux, les droits impliquent forcement les devoirs. "A force de parler chaque fois de droit à nos élèves, cela me pose un véritable problème. En tant enseignante, j'accorde plus d'importance aux devoirs et aux responsabilités de l'enfant. Si on y prend garde, l'enfant grandira avec l'idée qu'il n'a que des droits et ça c'est dangereux ».

Ils considèrent que les droits de l'enfant sont trop respectés, et que l'on devrait plutôt travailler sur les devoirs des enfants de nos jours vus les comportements déviants dans les écoles. Selon Desbiens J-F. et al. (2008), « la gestion de la classe s'effectue dans un contexte où l'école, à l'image de la société, doit composer avec la pluralité des valeurs morales et spirituelles de même qu'avec les répercussions des transformations de la vie et des rapports entre adultes et enfants au sein de la cellule familiale ». Les enseignants se retrouvent fréquemment alors confrontés à des difficultés pour gérer leur classe. Les deux courants de recherche qui se distinguent dans leur approche, permettent de comprendre les perceptions de ceux-là mêmes qui sont chargés de dispenser aux élèves les cours sur leurs droits en tant qu'enfants. D'un côté, l'approche s'intéressant à la structure des représentations sociales initialement développée par Abric (1976) puis par Flament, Guimelli (1998), Moliner et Rouquette (199). L'autre courant développé à la suite des travaux de Moscovici (1961), Doise (1990), Clémence et Lorenzi-Cioldi (1994) qui insistent sur les aspects différenciateurs des représentations sociales. Elles sont perçues comme étant «(...) des principes générateurs de prises de position liées à des insertions spécifiques dans un ensemble de rapports sociaux et organisant les processus symboliques intervenant dans ces rapports. » L'enseignement des droits des enfants n'étant pas toujours compris, il est 
nécessaire, de ne pas simplement parler de la Convention en tant que connaissance, mais surtout en tant que valeur importante au sein de la classe.

\section{Conclusion}

La nécessité d'intégrer la thématique des droits de l'enfant dans les classes afin d'ouvrir les esprits des élèves dès les premières années de leur scolarité, a conduit à s'intéresser au curriculum en vigueur dans le primaire. En se basant sur les guides maîtres, la prise en compte des thématiques relatives aux droits des enfants cadrent bien avec l'une des missions non négligeables de l'école qui est de former des élèves à la citoyenneté. Traiter cette thématique dans les classes, permettrait de développer l'esprit critique des élèves et également d'augmenter l'estime de soi chez ceux-ci considérés comme des « citoyens de demain ». L'enfant pourra alors se sentir partenaire à part entière et non pas un vulgaire « amasseur de connaissances ». Les instituteurs perçoivent différemment l'enseignement du module « droits des enfants » dans le contexte classe, laquelle décrite par Doyle " comme un environnement complexe caractérisé par la multidimentionnalité, la simultanéité, l'immédiateté, l'imprévisibilité, l'accessibilité ainsi que l'historicité des événements qui y surviennent » (cité par J-F Desbiens et al., 2009, p.180) ${ }^{14}$. Or, comme l'avait déjà noté Emile Durkheim (1992, 1ère ed. 1922) qu'il est appris à l'école plus d'éléments, en faisant allusion au système de règles liées à la forme scolaire qui concourt à inculquer l'esprit de discipline chez l'enfant. Il décrit donc un système d'éducation morale qui construit un système de valeur donné. La prise en compte des droits des enfants dans les contenus enseignés participent à la construction de système de valeur, qui constitue un référentiel pour les enfants. Toutefois, une formation et renforcement de capacités seront nécessaire pour briser les schèmes.

\section{References:}

1. Abric, J.C. (1976). «Jeux, conflits et représentations sociales » (thèse de doctorat non publiée). Université Aix en Provence, France.

2. Alpe, Y. (2006). "Quelle est la légitimité des savoirs scolaires", in Legardez A. \& Simmoneaux L. [coord.], L'école à l'épreuve de l'actualité. Enseigner les questions vives. Paris : ESF, p. 233-246.

3. Apple, M. W. (2004). Ideology and Curriculum, London: Routledge \& Kegan Paul.

4. Desbiens, J-F., Lanoue, S., Turcotte, S., Tourigny, J-S. \& Spallanzani, C. (2009). «Perception de la fréquence d'apparition des comportements perturbateurs par des stagiaires en enseignement de

\footnotetext{
${ }^{14}$ Perception de la fréquence d'apparition des comportements perturbateurs par des stagiaires en enseignement de l'éducation physique et à la santé (EPS). Nouveaux cahiers de la recherche en éducation, 12(2), 179-193. https://doi.org/10.7202/1017466
} 
l'éducation physique et à la santé (EPS) » Nouveau cahier de la recherche en éducation, vol. 12, no 2, 2009, p. 179 à 193.

5. Doise, W. \& Lorenzi-Cioldi, F. (1994). Prise de position et principes organisateurs des représentations sociales. In $\mathrm{Ch}$. Guimelli(dir.), Structures et transformations des représentations sociales. Lausanne : Delachaux \& Niestlé

6. Doise, W. (1990). Les représentations sociales. In C. Bonnet, R. Ghiglione, \& T.F. Richard.

7. Document réalisé par l'UNICEF Grande-Bretagne, en s'appuyant sur l'initiative britannique, Rights Respecting Schools Award : http://www.unicef.org.uk/Education/

8. Doyle, W. (1986). Classroom organization and management. In M.C. Wittrock (dir.), Handbook of research on teaching (p. 392-431). New York, NY : Macmillan.

9. Durkheim, E. (1992). Éducation et sociologie. Paris, PUF

10. Forquin, J. (1990). Sociologie de l'éducation, dix ans de recherche. Paris : INRP/L'Harmattan.

11. Guides maître de l'éducation nationale et de l'alphabétisation des différents niveaux (CP, CE et CM), année scolaire 2020-2021.

12. Lansdown, G. (2010). The realisation of children's participation rights : critical reflections. In B. Percy-Smith \& N. Thomas (éds.), $A$ handbook of children and young people participation: Perspectives from theory and practices (p. 11-23). Londres, Royaume-Uni : Routledge.

13. Lansdown, G. (2001). Promouvoir la participation des enfants au processus décisionnel démocratique. Florence, Italie: UNICEF.

14. Moliner, P. (1994). «Les méthodes de repérage et d'identification du noyau des représentations sociales. » In: C. Guimelli (Ed.), Structures et transformations des représentations sociales (pp. 199-232). Neuchâtel : Delachaux et Niestlé

15. Moscovici, S. (1961). La psychanalyse, son image et son public. Paris : PUF.

16. Nozaki, Y. \& Apple, W. (2002). « Ideology and curriculum », in: Levinson, D. L., Cookson P.

17. Perrenoud, P. (1993). Curriculum : «le formel, le réel, le caché ». In Houssaye, J. (dir.) La pédagogie : une encyclopédie pour aujourd'hui. Paris : ESF, pp. 61-76.

18. Rapport sur la mise en œuvre de la Convention et de la Recommandation concernant la lutte contre la discrimination dans le domaine de l'enseignement (juin 2016). 
19. Tisdall, E.K.M. (2015b). Addressing the challenges of children and young people's participation: Considering time and space. In T. Gal \& B.F. Duramy (éds.), Promoting the participation of children and young across the globe: From social exclusion to child-inclusive policies (p. 381-404). Oxford, Royaume-Uni : Oxford University Press.

20. Zermatten, J. (2004). Informations complémentaires par Jean Zermatten. In J. Zermatten (Ed.), Les droits de l'Enfant : Douze récits pour ne pas s'endormir, IDE. (pp. 189-267). Saint-Maurice : Editions Saint-Augustin.

21. https://fr.unesco.org/news/ce-que-vous-devez-savoir-droit-leducation 